\title{
Coordination Variables and Consensus Building in Multiple Vehicle Systems
}

Tim McLain

Brigham Young University - Provo, mclain@byu.edu

Follow this and additional works at: https://scholarsarchive.byu.edu/facpub

Part of the Mechanical Engineering Commons

\section{Original Publication Citation}

Ren, W., Beard, R., and McLain, T. Coordination Variables and Consensus Building in Multiple Vehicle Systems, in Cooperative Control, Springer-Verlag Series: Lecture Notes in Control and Information Sciences (V. Kumar, N.E. Leonard, and A.S. Morse, eds.), vol. 309, 2004.

\section{BYU ScholarsArchive Citation}

McLain, Tim, "Coordination Variables and Consensus Building in Multiple Vehicle Systems" (2004). Faculty Publications. 1907.

https://scholarsarchive.byu.edu/facpub/1907 


\title{
Coordination Variables and Consensus Building in Multiple Vehicle Systems
}

\author{
Wei Ren, Randal W. Beard, and Timothy W. McLain \\ Brigham Young University, Provo, Utah 84602 \\ $\{$ weiren, beard $\}$ @ee. byu.edu, mclain@byu.edu
}

Much of the research focus in the cooperative control community has been on formation control problems $[1,3,7,10,19]$. This focus may be due to the fact that the group control problem can be reduced to well-established single-agent control problems by employing a leader-follower type control strategy. For example, single-agent path planning and trajectory generation techniques can be employed for the leader, and conventional trajectory tracking strategies can be employed for the followers. Indeed, formation control problems are much like linear systems theory: we search where the light is the brightest. It can be argued that formation control problems are the simplest type of coordination problems and that even if they were to be completely solved, the solution would be of limited usefulness since the formation concept is of limited utility. This last comment is supported by the observation that humans cooperate to perform a wide variety of tasks, yet we rarely maintain formation with each other.

The usefulness of cooperative control technologies will be greatly enhanced if, as a community, we develop techniques that apply more generally to nonformation cooperative control problems. The first requirement is that we understand the fundamental issues inherent in all coordination problems. Toward that end, we offer the following, intuitively appealing, fundamental axiom:

Axiom 1 Shared information is a necessary condition for coordination.

Underlying this axiom are two important questions: "What information should be shared?" and "With whom should information be shared?" The focus of this chapter is on providing answers to these two questions.

In every cooperative control problem, there must be an identifiable cooperation objective. To achieve the team objective, specific kernels of information must be shared among members of the team. Identification of the key pieces of information to be shared is a critical step in the formulation of a cooperative control solution. Our approach is to collect the information that must be jointly shared to facilitate cooperation into a vector quantity called the coor- 
dination variable. The coordination variable represents the minimal amount of information needed to effect a specific coordination objective.

Although it is known by different names, the notion of a coordination variable is found in many other works on cooperative control. For example [16, 17] introduce an "action reference" which, if known by each vehicle, facilities formation keeping. In leader-following applications [25, 27], the states of the leader constitute the coordination variable since the actions of the other vehicles in the formation are completely specified once the leader states are known. In $[3,18,19]$, the notion of a virtual structure is used to derive formation control strategies. The motion of each vehicle is causally dependent on the dynamic states of the virtual structure, therefore the states of the virtual structure are the coordination variables. In [26] a team of autonomous underwater vehicles are controlled to swarm around a desired mean location of the team with a specified standard deviation. The action of each vehicle is dependent on the location of its nearest neighbor, and the desired mean and standard deviation. This information is the coordination variable.

Coordination variables may also be more discrete in nature. For example, in $[6,23]$, cooperative task allocation is addressed. Individual vehicle behavior is dependent on the task allocation vector which becomes the coordination variable. Similarly, in [11], the coordination variable is the dynamic role assignment in a robot soccer scenario.

Information necessary for cooperation may be shared in a variety of ways. For example, relative position sensors may enable vehicles to construct state information for other vehicles [8], or knowledge may be communicated between vehicles using a wireless network [12], or joint knowledge might be pre-programmed into the vehicles before a mission begins [2]. In Section 1 we offer some definitions and general principles regarding coordination variables.

For cooperative control strategies to be effective, a team of vehicles must be able to respond to unanticipated situations or changes in the environment that are sensed as a cooperative task is carried out. As the environment changes, the vehicles on the team must be in agreement as to what changes took place. A direct consequence of Axiom 1 is that cooperation requires that the group of agents reach a consensus on the coordination data. In other words, the instantiation of the coordination variable on each agent must asymptotically approach a sufficiently common value.

A critical problem for cooperative control is to determine algorithms so that a team of vehicles can reach consensus on the values of the coordination data in the presence of (1) imperfect sensors, (2) communication dropout, (3) sparse communication topologies, and (4) noisy and unreliable communication links. The question of "With whom does communication take place?" is of great significance in seeking consensus among a team of vehicles. Section 2 states some results on multi-agent consensus seeking for fixed communication topologies.

The consensus problem has recently been addressed in $[9,15,12]$. The work reported in [15] is particularly relevant to the results reported in this paper. 
Ref [15] addresses the knowledge consensus problem when teams of agents only have local communication between nearest neighbors. Since the set of nearest neighbors is constantly changing, the overall system becomes a hybrid system. The paper shows that if the union over all bidirectional communication graphs is connected for finite periods of time, then consensus is achieved. While the results in this paper are not as strong, only unidirectional communication links are assumed.

\section{Coordination Variables and Functions}

This section introduces a general approach to coordination problems where the team objectives are coupled through the assigned tasks rather than through dynamic interactions or tight physical constraints.

Cooperative control by a team of vehicles is dependent on the environment or mission scenario in which the vehicles are acting. To characterize the significant elements of the environment, define $\mathcal{X}_{i}$ to be the situation state space for the $i^{\text {th }}$ vehicle and let $\mathbf{x}_{i} \in \mathcal{X}_{i}$ be the situation state of the $i^{\text {th }}$ vehicle. For many cooperation problems, the situation state would include current information about an agent's position and the environment in which the agent is acting. For a given situation $\mathbf{x}_{i}$, the set of feasible actions for an agent is given by $\mathcal{U}_{i}\left(\mathbf{x}_{i}\right)$ and $\mathbf{u}_{i} \in \mathcal{U}_{i}$ is the action variable for the $i^{\text {th }}$ agent. The choice of the action variable by each agent on the team affects both the feasibility and the quality of the cooperation achieved.

Axiom 1 implies that there is a minimum amount of information needed by the team to effect cooperation. We will call this information the coordination variable and denote it by $\theta$. The essential idea is that if every agent knows the coordination variable and responds appropriately, then cooperative behavior will be achieved. The coordination variable is a vector in coordination space $\mathbb{R}^{c}$.

A representation of the distillation of information from the situation state and influence variables (full information) to the coordination variable (minimal information) is central to this method. If $f_{i}: \mathcal{X}_{i} \times \mathcal{U}_{i} \rightarrow \mathbb{R}^{c}$ is a function that maps situation state and influence vector pairs to $\mathbb{R}^{c}$, then the set of feasible coordination variables for the $i^{\text {th }}$ vehicle at state $\mathbf{x}_{i}$ is given by

$$
\Theta_{i}\left(\mathbf{x}_{i}\right)=\bigcup_{\mathbf{u}_{i} \in \mathcal{U}_{i}\left(\mathbf{x}_{i}\right)} f_{i}\left(\mathbf{x}_{i}, \mathbf{u}_{i}\right)
$$

Note that $\Theta_{i}\left(\mathbf{x}_{i}\right)$ is not necessarily a connected set.

We assume that $f_{i}$ is (pseudo) invertible in the sense that there exists a function $f_{i}^{\dagger}: \mathcal{X}_{i} \times \Theta_{i} \rightarrow \mathcal{U}_{i}$ (called the pseudo-inverse of $f$ ), such that for every $\vartheta \in \Theta_{i}\left(\mathbf{x}_{i}\right), f_{i}\left(\mathbf{x}_{i}, f_{i}^{\dagger}\left(\mathbf{x}_{i}, \vartheta\right)\right)=\vartheta$. Simply stated, if the situational state and the coordination variable are known, the decision variable is unique.

In addition to cooperative behavior, the team may have individual performance objectives. Associated with the $i^{\text {th }}$ vehicle is a myopic performance 
objective $J_{i}: \mathcal{X}_{i} \times \mathcal{U}_{i} \rightarrow \mathbb{R}$ that is assumed to be in harmony with the team objectives. The myopic cost can be parameterized as a function of the coordination variable. This can be done by using the relationship $\mathbf{u}_{i}=f_{i}^{\dagger}\left(\mathbf{x}_{i}, \vartheta\right)$, for each $\vartheta \in \Theta_{i}\left(\mathbf{x}_{i}\right)$. The function

$$
\phi_{i}\left(\mathbf{x}_{i}, \vartheta\right)=J_{i}\left(\mathbf{x}_{i}, f_{i}^{\dagger}\left(\mathbf{x}_{i}, \vartheta\right)\right),
$$

is a representation of the local myopic cost $J_{i}\left(\mathbf{x}_{i}, \mathbf{u}_{i}\right)$. Under the restriction that for each $\vartheta \in \Theta_{i}$ there is a unique $\mathbf{u}_{i} \in \mathcal{U}_{i}, \bigcup_{\vartheta \in \Theta_{i}\left(\mathbf{x}_{i}\right)} \phi_{i}\left(\mathbf{x}_{i}, \vartheta\right)=$ $\bigcup_{\mathbf{u}_{i} \in \mathcal{U}_{i}\left(\mathbf{x}_{i}\right)} J_{i}\left(\mathbf{x}_{i}, \mathbf{u}_{i}\right)$. If this is not the case, it follows that $\bigcup_{\vartheta \in \Theta_{i}\left(\mathbf{x}_{i}\right)} f_{i}^{\dagger}\left(\mathbf{x}_{i}, \vartheta\right)$ may only be a proper subset of $\mathcal{U}_{i}$, and $\phi_{i}\left(\mathbf{x}_{i}, \cdot\right)$ an approximation of $J_{i}\left(\mathbf{x}_{i}, \cdot\right)$. The function

$$
\phi_{i}: \mathcal{X}_{i} \times \Theta_{i}\left(\mathbf{x}_{i}\right) \rightarrow \mathbb{R}
$$

given by Equation (2) is called the coordination function of the $i^{\text {th }}$ vehicle. For a given situation state $\mathbf{x}_{i}$, the coordination function parameterizes the myopic cost of the $i^{\text {th }}$ vehicle verses the coordination variable.

In this chapter, the cooperation problems of interest can be posed as a minimization of a team objective function, where the team objective is a function of the myopic objective functions. Let $\mathcal{J}_{T}: \mathbb{R}^{N} \rightarrow \mathbb{R}$ be the team objective function, then the cooperative control problem is to find influence variables $\mathbf{u}_{1}, \ldots \mathbf{u}_{N}$ that solve the following optimization problem:

$$
\left(\mathbf{u}_{1}, \ldots, \mathbf{u}_{N}\right)=\arg \min _{\mathcal{U}_{1} \times \cdots \times \mathcal{U}_{N}} \mathcal{J}_{T}\left(J_{1}\left(\mathbf{x}_{1}, \mathbf{u}_{1}\right), \cdots, J_{N}\left(\mathbf{x}_{N}, \mathbf{u}_{N}\right)\right)
$$

subject to

$$
f_{i}\left(\mathbf{x}_{i}, \mathbf{u}_{i}\right)=f_{j}\left(\mathbf{x}_{j}, \mathbf{u}_{j}\right), \quad \forall i, j \in\{1, \cdots, N\} .
$$

This optimization problem will clearly pose computational problems as the number of vehicles increase, and for large states and influence dimensions.

Using coordination variables and coordination functions, a decomposition of the optimization problem of Equations (3) and (4) that captures the information essential for cooperation can be posed:

$$
\theta=\arg \min _{\vartheta \in \cap \Theta_{i}\left(\mathbf{x}_{i}\right)} \mathcal{J}_{T}\left(\phi_{1}(\vartheta), \cdots, \phi_{N}(\vartheta)\right)
$$

Once a team optimal value for the coordination variable is found, individual vehicle decisions can be found by solving for the influence variable from the relationship

$$
\mathbf{u}_{i}=f_{i}^{\dagger}\left(\mathbf{x}_{i}, \theta\right)
$$

This two-level decomposition process significantly reduces the computation and communication loads.

Coordination variables and functions have been applied successfully to UAV cooperative timing missions $[21,20]$ and UAV cooperative reconnaissance problems [4]. An illustrative example is given in the next section. 


\subsection{Example: Cooperative Timing}

The application of coordination variables and functions can be demonstrated by a simple example. Suppose that a group of friends decides that they will meet for dinner on a certain date, but fail to specify a specific time and place. On the afternoon of the dinner date, everyone realizes that they are uncertain about where or when to meet for dinner. For the moment, assume that all of the friends can get together on a conference call to make a decision. This group cooperation problem can be used to illustrate the cooperation strategy outlined above.

Clearly the coordination variables that must be determined for the group are the restaurant to eat at and the time to meet. For each individual there is range of feasible times and places to meet described by $\Theta_{i}$. This range is determined by the situation state $\mathbf{x}_{i}$ (e.g., traffic conditions, work location) and by the individual's actions $\mathbf{u}_{i}$ (e.g., departure time, choice to change clothes, choice of route).

The coordination function describes the cost to each individual over the range of the coordination variable. Influencing the cost might be the distance to the restaurant, the budget of the individual, the dietary tastes or restrictions of the individual, or the average wait-time at the restaurant. Thus, for every $\vartheta \in \Theta_{i}$, the coordination function $\phi_{i}\left(\mathbf{x}_{i}, \vartheta\right)$ describes the feeling of the individual about all of the possible choices of time and place in the form of a numeric cost metric.

In the conference call, coordination functions are exchanged and a decision can be made about the restaurant and meeting time that maximizes the collective happiness of the group. Although the happiness of each individual is unlikely to be maximal, the goal would be to craft the group objective function so that all of the friends are satisfied with the decision. Note that the exchange of information is efficient. The coordination function captures the essential information necessary to come to a group decision. No discussion of work load, dietary tastes, personal finances, or travel routes is necessary. Furthermore, once a group decision for the coordination variable $\theta$ is made, all individual decisions, such as those about the route and departure time, are left to the individual. This decomposition of group and individual decisions streamlines the cooperation process leading to both efficient decision making and communication.

In this example, the requirement that each agent exchange coordination function information with every other agent is limiting. Is it be possible to come to a decision by placing phone calls among individuals? If individual members of the group are not in agreement about the situation state (e.g., a traffic accident on a main thoroughfare or the availability of seating at a restaurant), is coming to an agreement on time and place possible? The following section deals specifically with the questions of who must communicate and how differences in information among members of the team can be resolved. 


\section{Consensus Seeking}

Coordination variables focus the attention on the minimal amount of information required for cooperation. The essential idea is that if each vehicle has the same instantiation of the coordination variable, then team action will be coordinated provided that each vehicle acts to achieve the desired value of the coordination variable. The consensus problem is to ensure that there is a sufficiently common instantiation of the coordination variable among members of the team. As shown in Figure 1, consensus can be formed on the situational state $\mathbf{x}_{i}$, or the coordination variable $\theta_{i}$. To be general, we will let $\xi_{i}$ represent the $i^{\text {th }}$ vehicle's "information" variable over which consensus is to be formed.

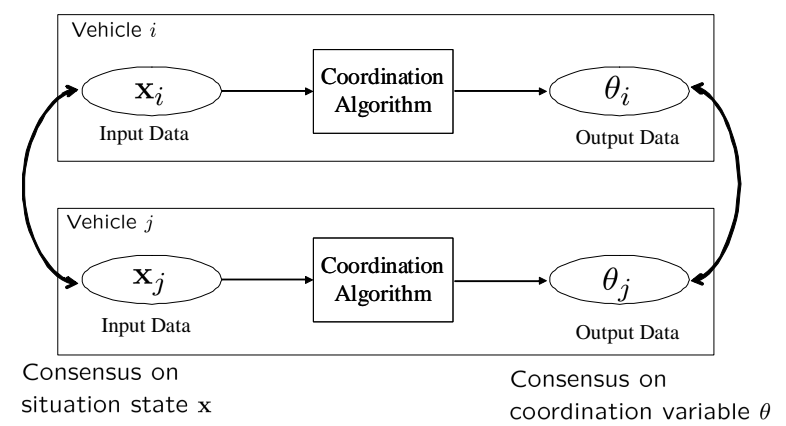

Fig. 1. Consensus Diagram.

Let $\mathcal{G}$ be a directed graph $\mathcal{G}$ (c.f. [13]) representing the (possibly unidirectional) communication topology, where vertices, denoted $A_{i}, i=1, \cdots, N$, represent the vehicles and edges represent unidirectional communication links between vehicles. A directed tree is a directed graph, where every vertex, except the root, has exactly one parent. A spanning tree of a directed graph is a tree formed by graph edges that includes all the vertices of the graph. We assume unidirectional communication to allow for scenarios where some of the agents do not possess a transmitter, or perhaps do not wish to transmit information, either to conserve energy or to increase stealth.

The linear consensus scheme proposed in [5] is

$$
\dot{\xi}_{i}=-\sum_{j=1}^{N} k_{i j} G_{i j}\left(\xi_{i}-\xi_{j}\right), \quad i=1, \cdots, N,
$$

where $k_{i j}$ are positive constants, and $G_{i j}$ is 1 if information flows from $A_{j}$ to $A_{i}$, and 0 otherwise. The intuition behind Equation (5) is that when $\mathcal{A}_{i}$ receives information from $\mathcal{A}_{j}$, its information variable is "pushed" toward $\mathcal{A}_{j}$ 's information variable with strength $k_{i j}$.

In the case of $\xi_{i} \in \mathbb{R}$, Eq. (5) can be written in matrix form as 


$$
\dot{\xi}=C \xi,
$$

where $\xi=\left[\xi_{1}, \cdots, \xi_{N}\right]^{T}, C=\left[c_{i j}\right],(i, j)=1, \cdots, N$, with $c_{i i}=-\left(\sum_{j \neq i} k_{i j} G_{i j}\right)$, $i=1, \cdots, N$, and $c_{i j}=k_{i j} G_{i j}, j \neq i$.

We say that $C$ is the matrix associated with graph $\mathcal{G}$. Note that this update scheme accommodates all possible communication topologies. In [5] the information variable $\xi_{i}$ is assumed to be a scalar and continuously differentiable in time. For simplicity, we maintain this assumption, but note that all the results are valid for $\xi_{i} \in \mathbb{R}^{p}$ by simply multiplying each element of $C$ by an identity matrix $I_{p}$ so that matrix $C$ has a dimension $N p \times N p$ instead of $N \times N$. In [5] the weightings $k_{i j}$ are assumed to be equal. In this chapter, we relax this assumption by allowing $k_{i j}$ to be any positive constant representing the relative confidence between vehicles. We will assume that the graph $\mathcal{G}$ is time-invariant.

We have the following definition from [5].

Definition 1. The set of agents $\mathcal{A}=\left\{A_{i} \mid i=1, \cdots, N\right\}$ is said to be in consensus at time $t_{0}$, if $t \geq t_{0}$ implies that $\left\|\xi_{i}(t)-\xi_{j}(t)\right\|=0$ for each $(i, j)=$ $1, \ldots, N$. The set of agents $\mathcal{A}$ is said to reach global consensus asymptotically if for any $\xi_{i}(0), i=1, \ldots, N,\left\|\xi_{i}(t)-\xi_{j}(t)\right\| \rightarrow 0$ as $t \rightarrow \infty$ for each $(i, j)=$ $1, \ldots, N$. The set $\mathcal{A}$ is said to be global consensus reachable if there exists an information update strategy for each $\xi_{i}, i=1, \ldots, N$ that achieves global consensus asymptotically for $\mathcal{A}$.

Obviously $C$ is diagonally dominant, has zero row sum, and non-positive diagonal elements. Therefore, from the Gersgorin disc theorem (c.f. [14]), $C$ has at least one zero eigenvalue and all the other non-zero eigenvalues are in the open left half plane.

\subsection{Consensus and Evolution of Coordination Variables}

In this section, we first consider the case when the information variable is inherently constant. We then consider the case when the information variable is dynamically evolving in time. This is the case, for example, in formation control problems where the information variable is the dynamic state of a virtual leader.

\section{Static Consensus}

It has been shown in [5] that the group of vehicles $\mathcal{A}$ reach consensus asymptotically using the update scheme (5) if matrix $C$ in Eq. (6) has exactly one zero eigenvalue and all the others are in the open left half plane. The following result computes the value of the information variable that is reached through the consensus process.

Before moving on, we need the following definitions from matrix theory (c.f. [14]). A real matrix $M=\left[a_{i j}\right]$ is said to be nonnegative, denoted as 
$M \geq 0$, if all its entries are nonnegative. A nonnegative matrix is said to be a stochastic matrix if all its row sums are 1 .

Lemma 1. If $C$ is given by Eq. (6), then $e^{C t}, \forall t>0$, is a stochastic matrix with positive diagonal entries. Furthermore, if $C$ has exactly one zero eigenvalue, then $e^{C t} \rightarrow b \nu^{T}$ and $\xi_{i}(t) \rightarrow \sum_{i=1}^{N}\left(\nu_{i} \xi_{i}(0)\right)$ as $t \rightarrow \infty$, where $b=[1, \cdots, 1]_{N \times 1}^{T}, \nu=\left[\nu_{1}, \cdots, \nu_{N}\right]^{T} \geq 0$, and $\sum_{i=1}^{N} \nu_{i}=1$.

Proof: Given eigenvalues $\lambda_{i} \in \sigma(C)$ with eigenvectors $z_{i}, i=1, \cdots, N$, where $\sigma(A)$ represents the spectrum of $A$, we know that $e^{\lambda_{i} t} \in \sigma\left(e^{C t}\right)$ with the same eigenvectors as $C$ (c.f. [14]). Noting that $C$ has a zero eigenvalue with an associated eigenvector given by $b$, then $e^{C t}$ has an eigenvalue 1 with the same eigenvector $b$. Thus we know that $e^{C t} b=b$, which implies that $e^{C t}$ always has row sum equal to 1 . Also note that $C$ can be written as the sum of a nonnegative matrix $M$ and $-\beta I_{N}$, where $\beta$ is the maximum absolute value of the diagonal entries of $C$ and $I_{N}$ is the $N \times N$ identity matrix. We can see that $e^{C t}=e^{-\beta t} e^{M t}$, which is obviously nonnegative and has positive diagonal entries. As a result, $e^{C t}, \forall t>0$, is a stochastic matrix with positive diagonal entries.

Furthermore, if $C$ has exactly one zero eigenvalue, then $e^{C t}$ has exactly one eigenvalue equal to 1 and all the other eigenvalues have modulus less than 1. Let $J=\left[j_{m l}\right],(m, l)=1, \cdots, N$, be the Jordan matrix corresponding to matrix $C$, then $j_{m m}=\lambda_{m}$. Without loss of generality, assume that $\lambda_{N}=0$ and $\lambda_{m}$ is on the open left half plane, $m=1, \cdots, N-1$.

Let $C=P J P^{-1}$, where $P=\left[p_{1}, \cdots, p_{N}\right]$ is an $N \times N$ matrix. Note that $p_{N}$ can correspond to an eigenvector associated with eigenvalue $\lambda_{N}=0$. Without loss of generality, choose $p_{N}=b$ as the eigenvector.

We know that $e^{C t}=P e^{J t} P^{-1}$. It can be verified that

$$
e^{J t} \rightarrow\left[\begin{array}{cccc}
0 & 0 & \cdots & 0 \\
\vdots & \vdots & \ddots & \vdots \\
0 & 0 & \cdots & 0 \\
0 & 0 & \cdots & 1
\end{array}\right]
$$

as $t \rightarrow \infty$ from the property of $C$ (c.f. [14]). After some manipulation, we know that $e^{C t} \rightarrow b \nu^{T}$ as $t \rightarrow \infty$, where $\nu_{i}, i=1, \cdots, N$, corresponds to the last row of matrix $P^{-1}$. The result $\sum_{i=1}^{N} \nu_{i}=1$ comes from the fact that $e^{C t}$ has row sum equal to 1 for any $t$.

We also need to show that $\nu \geq 0$. Now consider matrix $e^{C k}, k=0,1,2, \cdots$. Obviously $e^{C k}$ should also approach to $b \nu^{T}$ as $k \rightarrow \infty$. From Lemma 8.2.7 in [14], $\nu$ should be an eigenvector of matrix $\left(e^{C}\right)^{T}$ associated with the simple eigenvalue 1. From Theorem 8.3.1 in [14], $\left(e^{C}\right)^{T}$ has a nonnegative eigenvector $x \geq 0$ associated with the simple eigenvalue 1 . Thus it can be seen that $\nu=\alpha x$ for some $\alpha \neq 0$. Since $\sum_{i=1}^{N} \nu_{i}=1$, it must be true that $\alpha>0$, which implies that $\nu \geq 0$. 
The solution to Eq. (6) is given by $\xi(t)=e^{C t} \xi(0)$. Therefore, it is obvious that $\xi_{i}(t) \rightarrow \sum_{i=1}^{N}\left(\nu_{i} \xi_{i}(0)\right), i=1, \cdots, N$, as $t \rightarrow \infty$.

Note that if we replace matrix $C$ with $\gamma C$ in Eq. (6), where $\gamma>0$, we can increase consensus speed by increasing $\gamma$. The solution to Eq. (6) with this new matrix is given by $\xi=e^{\gamma C t} \xi(0)=e^{C(\gamma t)} \xi(0)$, which converges faster than the original solution if we choose $\gamma>1$.

Let $\mathcal{G}_{1}$ be a communication graph for the group of agents $\mathcal{A}$. Let $\mathcal{G}_{2}$ be the communication graph by adding one more directed link from any node $m$ to node $\ell$ to graph $\mathcal{G}_{1}$, where $m \neq l$. Also let $Q$ and $S$ be the matrices in the update law (6) associated with graphs $\mathcal{G}_{1}$ and $\mathcal{G}_{2}$ respectively. Denote $p_{Q}(t)=\operatorname{det}(t I-Q)$ and $p_{S}(t)=\operatorname{det}(t I-S)$ as the characteristic polynomial of $Q$ and $S$ respectively. Let $Q_{t}=t I-Q$ and $S_{t}=t I-S$. Given any matrix $M$, denote $M([i, j])$ as the sub-matrix of $M$ formed by deleting the $i$ th row and $j$ th column.

Lemma 2. If matrix $Q$ has exactly one zero eigenvalue, then so does matrix $S$.

Proof: Without loss of generality, we assume that the new directed communication link added to graph $\mathcal{G}_{1}$ is from node $m$ to node 1 , where $m \neq 1$, for simplicity since we can always renumber node $l$ as node 1 .

Obviously matrix $S$ has at least one zero eigenvalue and all the other nonzero eigenvalues are in the open left half plane. Below we will show that $S$ has only one zero eigenvalue.

Assume that $Q=\left[q_{i j}\right], S=\left[s_{i j}\right], Q_{t}=\left[q_{t i j}\right]$, and $S_{t}=\left[s_{t i j}\right],(i, j)=$ $1, \cdots, N$. From the property of $Q$ and $S$, we know that $s_{11}=q_{11}-k_{1 m}$, $s_{1 m}=q_{1 m}+k_{1 m}$, and $s_{i j}=q_{i j}$ otherwise. Accordingly, it can be seen that $s_{t 11}=t-s_{11}=t-q_{11}+k_{1 m}=q_{t 11}+k_{1 m}, s_{t 1 m}=-s_{1 m}=-q_{1 m}-k_{1 m}=q_{t 1 m}-$ $k_{1 m}$, and $s_{t i j}=q_{t i j}$ otherwise. Also note that $\operatorname{det} S_{t}([1, j])=\operatorname{det} Q_{t}([1, j])$, $j=1, \cdots, N$. Then we know that

$$
\begin{aligned}
\operatorname{det} S_{t}= & \sum_{j=1}^{N}(-1)^{1+j} s_{t 1 j} \operatorname{det} S_{t}([1, j]) \\
= & \sum_{j=1}^{N}(-1)^{1+j} q_{t 1 j} \operatorname{det} S_{t}([1, j]) \\
& +k_{1 m} \operatorname{det} S_{t}([1,1])-(-1)^{1+m} k_{1 m} \operatorname{det} S_{t}([1, m]) \\
= & \operatorname{det} Q_{t}+k_{1 m}\left(\operatorname{det} S_{t}([1,1])+(-1)^{m} \operatorname{det} S_{t}([1, m])\right) .
\end{aligned}
$$

Consider a matrix $E=\left[e_{i j}\right],(i, j)=1, \cdots, N-1$, given by adding $\left[s_{21}, s_{31}, \cdots, s_{N 1}\right]^{T}$ to the $(m-1)$ th column of matrix $S([1,1])$. Matrix $E$ can be denoted as 


$$
E=\left[\begin{array}{cccccc}
s_{22} & s_{23} & \cdots & s_{2 m}+s_{21} & \cdots & s_{2 N} \\
s_{32} & s_{33} & \cdots & s_{3 m}+s_{31} & \cdots & s_{3 N} \\
\vdots & \vdots & \vdots & \ddots & \vdots & \vdots \\
s_{N 2} & s_{N 3} & \cdots & s_{N m}+s_{N 1} & \cdots & s_{N N}
\end{array}\right]
$$

Thus $e_{i(m-1)}=s_{(i+1) m}+s_{(i+1) 1}, i=1, \cdots, N-1$. Using the properties of determinants, it can be verified that

$$
\operatorname{det}(t I-E)=\operatorname{det} S_{t}([1,1])+(-1)^{m} \operatorname{det} S_{t}([1, m]) .
$$

Obviously matrix $E$ has zero row sum and nonpositive diagonal elements. Also matrix $E$ is diagonally dominant. From the Gersgorin disc theorem, we know that $E$ has at least one zero eigenvalue and all the other non-zero eigenvalues are on the open left half plane. As a result, the Routh stability criterion implies that the characteristic polynomial of $E$ denoted as $\operatorname{det}(t I-E)$ has a nonnegative coefficient in the first power of $t$. We also know that matrix $Q$ has a positive coefficient for the first power of $t$ in its characteristic polynomial $\operatorname{det} Q_{t}$ since $Q$ has exactly one zero eigenvalue and all the others are in the open left half plane.

Noting that $\operatorname{det} S_{t}=\operatorname{det} Q_{t}+k_{1 m} \operatorname{det}(t I-E)$, it is obvious that $S$ has a positive coefficient for the first power of $t$.

Therefore, $S$ can only have one zero eigenvalue.

Ref. [5] shows that the group of agents $\mathcal{A}$ is global consensus reachable if and only if the associated communication graph $\mathcal{G}$ has a spanning tree. The proof for this claim in [5] is constructive in that the linear update law is based on a communication graph which is the spanning tree of $\mathcal{G}$. Of course, there may exist other connections in graph $\mathcal{G}$ which are ignored. Ref. [5] only partially answers the question of whether the update law (5) accounting for all existing connections achieves global consensus asymptotically. The next result provides a complete answer.

Theorem 2. The consensus strategy (5), achieves global consensus asymptotically for $\mathcal{A}$ if and only if the associated (static) communication graph $\mathcal{G}$ has a spanning tree.

Proof: (Sufficiency.) Obviously $C$ in Eq. (6) associated with graph $\mathcal{G}$ always has at least one zero eigenvalue and all the other non-zero eigenvalues are in the left half plane. We only need to check the algebraic multiplicity of the zero eigenvalue.

From [5], we know that the matrix associated with the spanning tree has exactly one zero eigenvalue. If graph $\mathcal{G}$ is itself the spanning tree, we know that the update law (5) achieves consensus asymptotically for $\mathcal{A}$. If not, graph $\mathcal{G}$ can be constructed by consecutively adding communication links to the tree. Lemma 2 implies that adding one additional communication link to the spanning tree results in an associated matrix that also has exactly one zero eigenvalue. We can recursively add additional links, where Lemma 2 implies 
that the matrix associated with the new graph has exactly one zero eigenvalue, until we obtain the graph $\mathcal{G}$. By induction, we know that the update law (5) achieves global consensus asymptotically for $\mathcal{A}$.

(Necessity.) The consensus strategy (5) achieves global consensus asymptotically for $\mathcal{A}$ implies that $\mathcal{A}$ is global consensus reachable, which in turn implies that graph $\mathcal{G}$ has a spanning tree following the necessity part of the proof for Theorem 3.1 in [5].

Corollary 1. Suppose that $B=\left[b_{i j}\right]$, where $b_{i i} \leq 0, b_{i j} \geq 0, \forall i \neq j$, and $\sum_{j=1}^{n} b_{i j}=0$. The $B$ has at least one zero eigenvalue and all the other nonzero eigenvalues are in the open left half plane. Furthermore, $B$ has exactly one zero eigenvalue if and only if the directed graph associated with $B$ has a spanning tree.

Proof: $B$ has the same property as matrix $C$ in Eq. (6), therefore the corollary follows from Theorem 2 .

Corollary 2. The Laplacian matrix of a graph has a simple zero eigenvalue if and only if the graph has a spanning tree.

Proof: If we multiply the Laplacian matrix by -1 , we get a matrix satisfying the properties defined in Corollary 1.

Note that the linear update law (5) only achieves consensus for constant coordination variables, which may not be suitable for applications where the coordination variable evolves dynamically. For example, in the context of leader-following approaches (c.f. [22]), the group leader's trajectory can act as the coordination variable for the whole group.

\section{Dynamic Consensus}

Suppose that the information variable on each vehicle is driven by the same time-varying input $u(t)$, which might represent an a priori known feedforward signal. The associated consensus scheme is given by

$$
\dot{\xi}_{i}=-\sum_{j=1}^{N} k_{i j} G_{j i}\left(\xi_{i}-\xi_{j}\right)+u(t), \quad i=1, \cdots, N .
$$

Eq. (7) can also be written in matrix form as

$$
\dot{\xi}=C \xi+B u(t)
$$

where $C$ is the matrix associated with graph $\mathcal{G}$ and $B=[1, \cdots, 1]^{T}$. We have the following theorem regarding consensus of the information variables $\xi_{i}$, $i=1, \ldots, N$. 
Theorem 3. The consensus strategy (8) achieves global consensus asymptotically for $\mathcal{A}$ if and only if the associated communication graph $\mathcal{G}$ has a spanning tree. Furthermore the information variables satisfy $\left\|\xi_{i}(t)-\zeta(t)\right\| \rightarrow 0$ as $t \rightarrow \infty$, where $\zeta(t)$ is the solution of

$$
\dot{\zeta}=u(t), \quad \zeta(0)=\mu,
$$

where $\mu B$ is equilibrium of the differential equation

$$
\dot{\pi}=C \pi, \quad \pi(0)=\xi(0) .
$$

Proof: (Sufficiency.) The solution to Eq. (8) is given by $\xi(t)=\xi_{s}(t)+\xi_{e}(t)$, where $\xi_{s}(t)=e^{C t} \xi(0)$ and $\xi_{e}(t)=\int_{0}^{t} e^{C \tau} B u(\tau) \mathrm{d} \tau$ (c.f. [24]). Note that $\xi_{s}$ represents the zero input solution to Eq. (8), that is, solution to $\dot{\xi}=C \xi$. From Theorem 2, it is obvious that each component of $\xi_{s}$ satisfies $\xi_{s i}(t) \rightarrow \mu$ as $t \rightarrow \infty, i=1, \cdots, N$. Also note that $\xi_{e}$ represents the zero state solution to Eq. (8). We know that $e^{C t} B=B$ since $e^{C t}$ always has row sum equal to 1 . Therefore, it can be seen that each component of $\xi_{e}$ satisfies $\xi_{e i}=\int_{0}^{t} u(\tau) \mathrm{dt}$, $i=1, \cdots, N$. Combining $\xi_{s}$ and $\xi_{e}$, gives $\left\|\xi_{i}(t)-\zeta(t)\right\| \rightarrow 0$ as $t \rightarrow \infty$.

(Necessity.) The necessary part follows directly from Theorem 2.

\section{Equilibrium Points}

We have shown that the linear consensus strategy (5) achieves global consensus asymptotically for $\mathcal{A}$ if the graph $\mathcal{G}$ has a spanning tree. In addition, $\xi_{i}(t)$ will converge to $\sum_{i=1}^{N}\left(\nu_{i} \xi_{i}(0)\right)$ as $t \rightarrow \infty$, where $\sum_{i=1}^{N} \nu_{i}=1$ and $\nu_{i} \geq 0$. A natural question is whether each initial condition $\xi_{i}(0)$ will contribute to the final equilibrium point. In the following we provide a partial answer to this question. We assume that graph $\mathcal{G}$ has a spanning tree in this section.

Observe that if there is a node $A_{k}$ in $\mathcal{G}$ without an incoming link (there is at most one such node in graph $\mathcal{G}$ from Theorem 2), the linear update law corresponding to this node is given by $\dot{\xi}_{k}=0$ from Eq. (5), which implies that $\xi_{k}(t)=\xi_{k}(0)$ for all $t$. Therefore, the other nodes must converge to $\xi_{k}(0)$ for any $k_{i j}>0$. That is, $\nu_{k}=1$ and $\nu_{i}=0, \forall i \neq k$.

In general, the initial condition of a node contributes to the equilibrium value if and only if the node has a directed path to all the other nodes in $\mathcal{G}$. Thus $\nu_{i} \neq 0$ for any node which has directed paths to all the other nodes in $\mathcal{G}$ and $\nu_{i}=0$ otherwise. As a special case, the initial condition of each node in a graph contributes to the final equilibrium point if and only if the graph is strongly connected. The above argument can be explained as follows. If there is no path from node $j$ to node $m$ in $\mathcal{G}$, it is impossible for $\xi_{m}(t)$ to be influenced by $\xi_{j}(0)$. On the other hand, if there is a path from node $j$ to every other node in $\mathcal{G}$, then $\xi_{i}(t), \forall i \neq j$, will be influenced by $\xi_{j}(0)$.

The fact that $\nu_{i} \geq 0, i=1, \cdots, N$ can also be explained from the following perspective. Assume that $\nu_{\ell}<0$ for some $\ell$. Consider the case $\xi_{\ell}(0)>0$ and 
$\xi_{i}(0)=0, \forall i \neq \ell$. We know that $\xi_{i}(t)$ will converge to $\sum_{i=1}^{N}\left(\nu_{i} \xi_{i}(0)\right)=\nu_{\ell} \xi_{\ell}(0)$, which is negative. Following the update law $(5), \dot{\xi}_{\ell}(0)<0$ if there is any incoming link to $A_{\ell}$ and $\dot{\xi}_{\ell}(0)=0$ otherwise. In the first situation, $\xi_{\ell}(t)$ will decrease and $\xi_{i}(t), \forall i \neq \ell$ cannot decrease since $\dot{\xi}_{i}(0) \geq 0$, which implies that $\xi_{i}(t)$ will be synchronized to a value $c$ with $0 \leq c<\xi_{\ell}(0)$. In the second situation, $\xi_{i}(t)$ will be synchronized to $\xi_{\ell}(0)$. Both cases are contradictory to the above result. Therefore, $\nu_{i} \geq 0, i=1, \cdots, N$.

\subsection{Illustrative Example}

In this section, we consider a scenario where six vehicles are to rendezvous at a position along a parameterized trajectory represented by $\left(r_{x}(\tau(t)), r_{y}(s(t))\right)$. Figure 2 shows the corresponding communication links between these vehicles. Note the existence of a spanning tree.

It is assumed that each vehicle knows the parameterized trajectory. Therefore the parameters $\tau$ and $s$ therefore represent the minimum information needed to achieve the coordination objective: i.e., $\tau$ and $s$ are the coordination variables. We will instantiate $\tau$ and $s$ on each vehicle as $\tau_{i}$ and $s_{i}$, $i=1, \cdots, 6$. Here we let $\xi_{i}=\left[\tau_{i}, s_{i}\right]^{T}, i=1, \cdots, 6$.

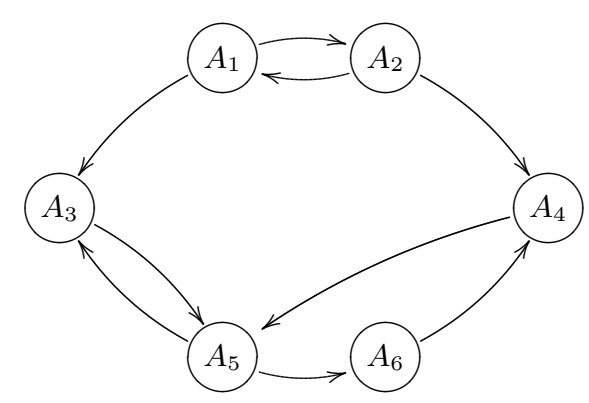

Fig. 2. Communication topology.

Based on the communication topology shown in Figure 2, the matrix $C$ is given by

$$
C=\gamma\left[\begin{array}{cccccc}
-1.5 & 1.5 & 0 & 0 & 0 & 0 \\
2 & -2 & 0 & 0 & 0 & 0 \\
0.9 & 0 & -2.8 & 0 & 1.9 & 0 \\
0 & 1.2 & 0 & -2.5 & 0 & 1.3 \\
0 & 0 & 1.4 & 1.8 & -3.2 & 0 \\
0 & 0 & 0 & 0 & 0.7 & -0.7
\end{array}\right] \otimes I_{2}
$$

where $\gamma>0$ is a coefficient, $\otimes$ denotes the Kronecker product, and $k_{i j}>0$, $(i, j)=1, \cdots, 6$, is chosen arbitrarily. The initial conditions for each instantiation of $\tau$ and $s$ are given by $\tau_{i}=0.2 i-0.1$ and $s_{i}=0.2 i, i=1, \cdots, 6$. 
Figure 3 shows the consensus scenario using update law (6) for $\gamma=1$ and $\gamma=5$ respectively. We can see that only the initial conditions of $A_{1}$ and $A_{2}$ affect the equilibrium value, which is consistent with the communication graph shown in Figure 2, where it can be seen that only $A_{1}$ and $A_{2}$ have a directed path to all the other nodes. Figure 4 shows the same consensus scenario corresponding to the communication graph formed by deleting the link from $A_{2}$ to $A_{1}$ in Figure 2. It can be seen that each instantiation of $\tau$ and $s$ converges to $\tau_{1}(0)$ and $s_{1}(0)$ respectively.
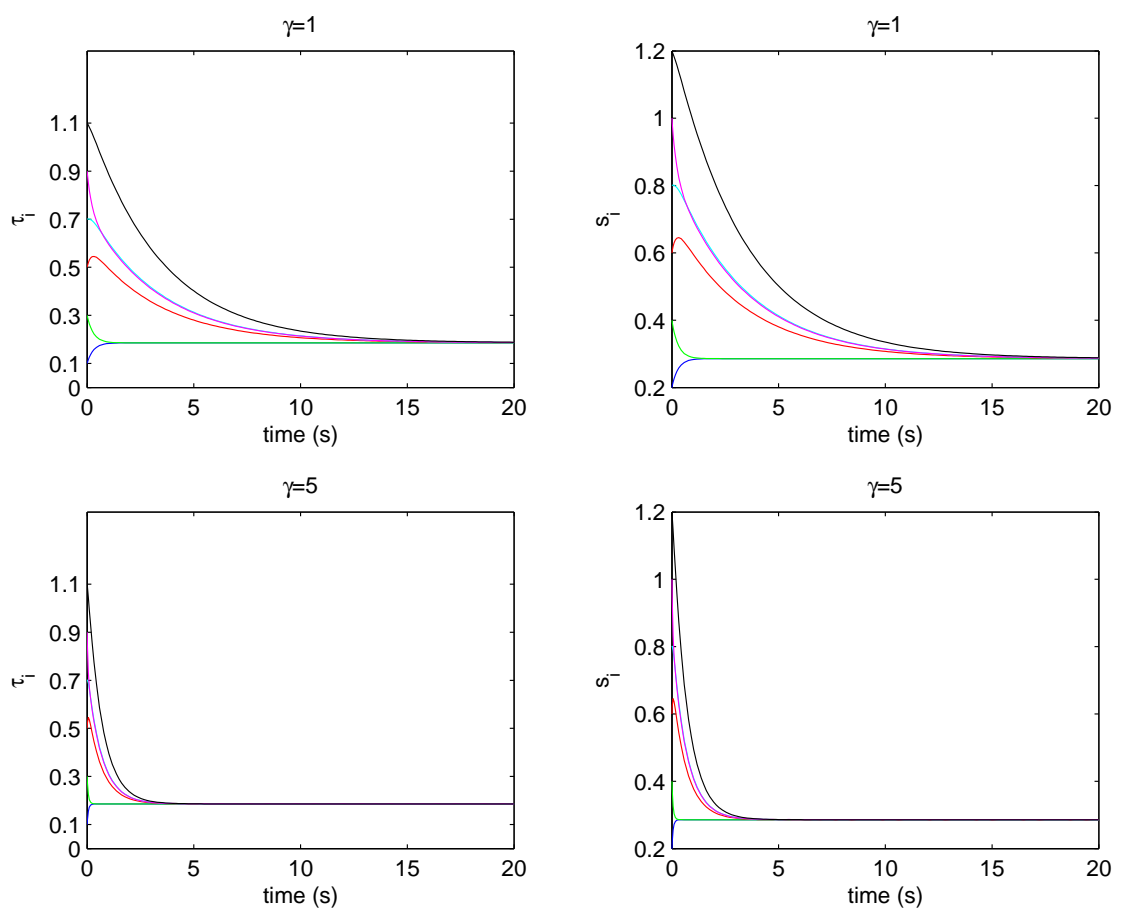

Fig. 3. Consensus of $\tau_{i}$ and $s_{i}$ using update law (6).

Figure 5 illustrates a dynamic consensus scenario using update law (8) for $\gamma=1$ and $\gamma=5$ respectively. The common predefined planning schemes for $\tau$ and $s$ are given by $\dot{\tau}=\frac{1}{5}|\sin (t)|$ and $\dot{s}=\frac{1}{4}|\cos (t)|$ respectively. Here we let $u(t)=\left[\frac{1}{5}|\sin (t)|, \frac{1}{4}|\cos (t)|\right]^{T}$ in Eq. (8). It can be seen that consensus is achieved asymptotically and that both $\tau_{i}$ and $s_{i}$ follow the appropriate trajectories. 

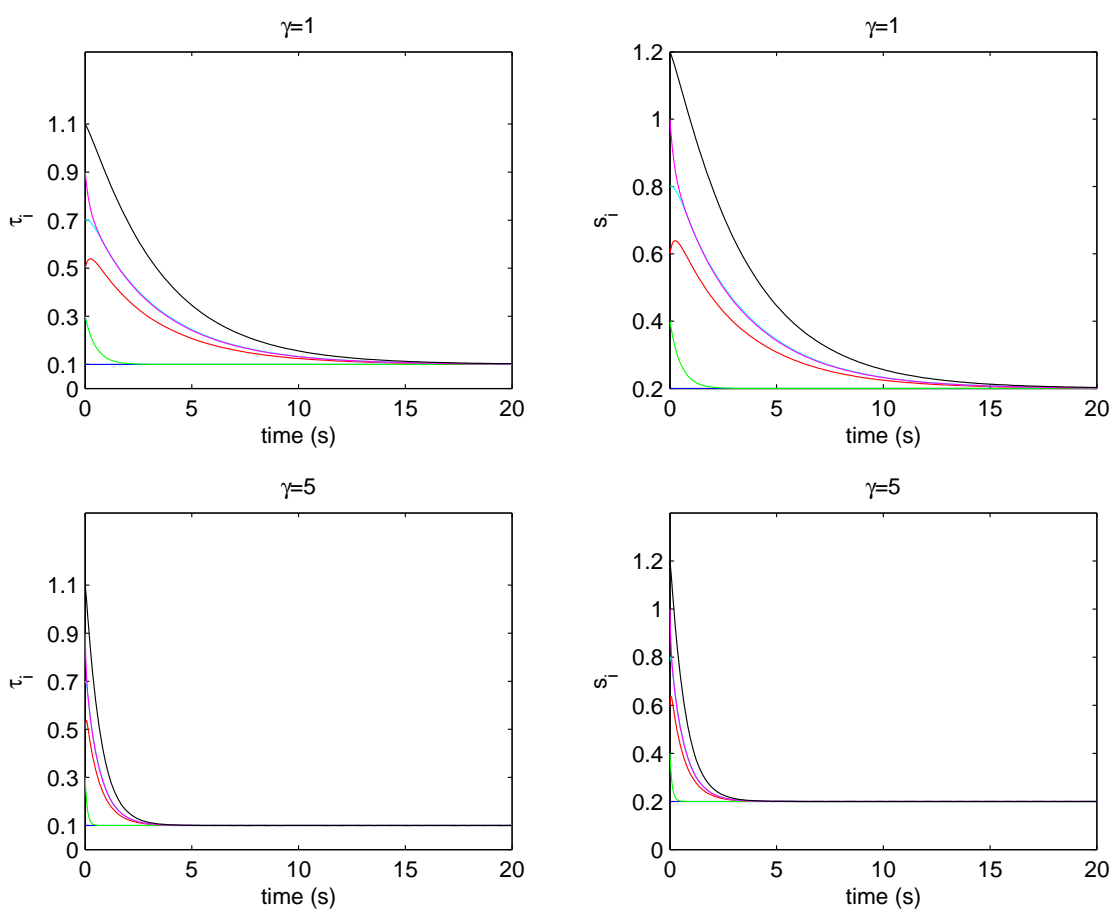

Fig. 4. Consensus of $\tau_{i}$ and $s_{i}$ without link from $A_{2}$ to $A_{1}$ using update law (6).

\section{Research Challenges and Future Directions}

In this chapter we have highlighted some of the challenging problems inherent in coordinated control. In particular, we have argued that coordination is inherently tied to information exchange. This perspective highlights several key problems that need to be addressed.

1. What are the appropriate coordination variables for broad classes of problems?

2. How does a group of vehicles form consensus on those variables when the data is (a) continuous in time, (b) discrete in time, (c) quantized in amplitude, or (d) originates from sources with variable reliability.

3. How do we make the team objectives invariant with respect to the consensus seeking problem? In other words, as consensus is being formed, the vehicles must act on the best information available to them at the time. One way of viewing this is that the individuals understand the team objectives differently. Under what conditions will the "design" objectives be satisfied? 

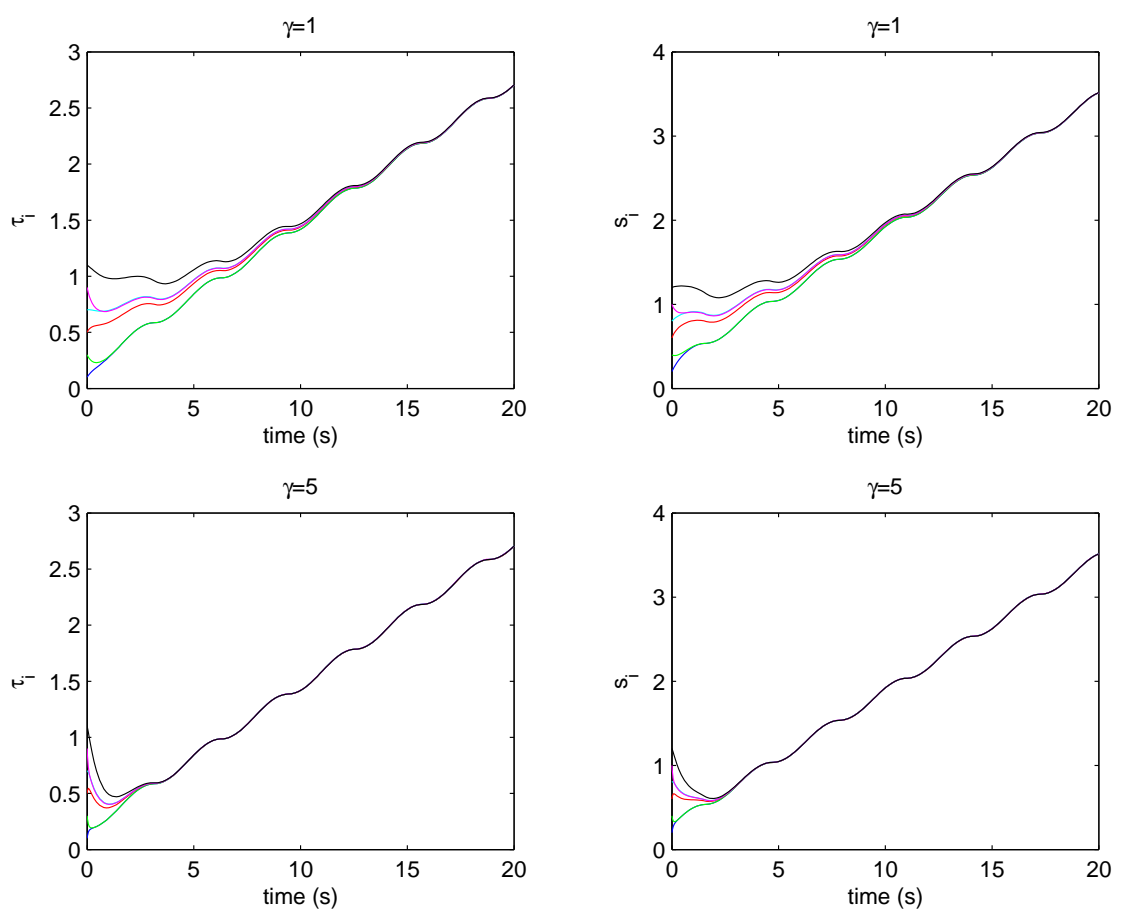

Fig. 5. Consensus and evolution of $\tau_{i}$ and $s_{i}$ using update law (8).

\section{Acknowledgements}

This work was partially funded by AFOSR grants F49620-01-1-0091 and F49620-02-C-0094, and by DARPA grant NBCH1020013.

\section{References}

1. Tucker Balch and Ronald C. Arkin. Behavior-based formation control for multirobot teams. IEEE Transactions on Robotics and Automation, 14(6):926-939, December 1998.

2. Tucker Balch and Lynne E. Parker, editors. Robot Teams: From Diversity to Polymorphism. A. K. Peters, Ltd., Natick, Massachusetts, 2002.

3. Randal W. Beard, Jonathan Lawton, and Fred Y. Hadaegh. A feedback architecture for formation control. IEEE Transactions on Control Systems Technology, 9(6):777-790, November 2001.

4. Randal W. Beard and Timothy W. McLain. Multiple UAV cooperative search under collision avoidance and limited range communication constraints. In Proceedings of the IEEE Conference on Decision and Control, 2003. To appear. 
5. Randal W. Beard and Vahram Stepanyan. Synchronization of information in distributed multiple vehicle coordinated control. In Proceedings of the IEEE Conference on Decision and Control, 2003. To appear.

6. John Bellingham, Michael Tillerson, Arthur Richards, and Johnathan P. How. Multi-task allocation and path planning for cooperating UAVs. In Cooperative Control: Models, Applications and Algorithms, pages 1-19. Conference on Coordination, Control and Optimization, November 2001.

7. Calin Belta and Vijay Kumar. Trajectory design for formations of robots by kinetic energy shaping. In Proceedings of the IEEE International Conference on Robotics and Automation, pages 2593-2598, Washington DC, May 2002.

8. J. Russell Carpenter. Decentralized control of satellite formations. International journal of Robust and Nonlinear Control, 12:141-161, 2002.

9. Gustavo Ayres de Castro and Fernando Paganini. Convex synthesis of controllers for consensus over a network of agents. In Proceedings of the IEEE Conference on Decision and Control, 2003. To appear.

10. Magnus Egerstedt and Xiaoming Hu. Formation constrained multi-agent control. IEEE Transactions on Robotics and Automation, 17(6):947-951, December 2001.

11. Rosemary Emery, Kevin Sikorski, and Tucker Balch. Protocols for collaboration, coordination and dynamic role assignment in a robot team. In Proceedings of the IEEE International Conference on Robotics and Automation, pages 3008-3015, Washington DC, May 2002.

12. J. Alexander Fax and Richard M. Murray. Graph laplacians and stabilization of vehicle formations. In IFAC World Congress, Barcelona, Spain, 2002.

13. C. Godsil and G. Royle. Algebraic Graph Theory, volume 207 of Graduate Text in Mathematics. Springer, New York, 2001.

14. Roger A. Horn and Charles R. Johnson. Matrix Analysis. Cambridge University, 1985.

15. Ali Jadbabaie, Jie Lin, and A. Stephen Morse. Coordination of groups of mobile autonomous agents using nearest neighbor rules. IEEE Transactions on Automatic Control, 48(6):988-1001, June 2003.

16. W. Kang, N. Xi, and Andy Sparks. Formation control of autonomous agents in 3D workspace. In Proceedings of the IEEE International Conference on Robotics and Automation, pages 1755-1760, San Francisco, CA, April 2000.

17. Wei Kang and Hsi-Han Yeh. Coordinated attitude control of mulit-satellite systems. International Journal of Robust and Nonlinear Control, 12:185-205, 2002.

18. Jonathan Lawton and Randal Beard. A projection approach to spacecraft formation attitude control. In 23rd Annual AAS Guidance and Control Conference, Breckenridge, Colorado, February 2000. Paper no. AAS 00-011.

19. Naomi Ehrich Leonard and Edward Fiorelli. Virtual leaders, artificial potentials and coordinated control of groups. In Proceedings of the IEEE Conference on Decision and Control, pages 2968-2973, Orlando, Florida, December 2001.

20. T. McLain, P. Chandler, S. Rasmussen, and M. Pachter. Cooperative control of UAV rendezvous. In Proc. of the ACC, pages 2309-2314, June 2001.

21. Timothy W. McLain and Randal W. Beard. Coordination variables, coordination functions, and cooperative timing missions. In Proceedings of the American Control Conference, pages 296-301, Denver, CO, June 2003. 
22. Wei Ren and Randal W. Beard. A decentralized scheme for spacecraft formation flying via the virtual structure approach. In Proceedings of the American Control Conference, pages 1746-1751, Denver, CO, June 2003.

23. A. Richards, J. Bellingham, M. Tillerson, and J. How. Coordination and control of UAVs. In Proceedings of the AIAA Guidance, Navigation, and Control Conference, pages AIAA-2002-4588, Monterey, CA, August 2002.

24. Wilson J. Rugh. Linear System Theory. Prentice Hall, Englewood Cliffs, New Jersey, 2nd edition, 1996.

25. Shahab Sheikholeslam and Charles A. Desoer. Control of interconnected nonlinear dynamical systems: The platoon problem. IEEE Transactions on Automatic Control, 37(6):806-810, June 1992.

26. Daniel J. Stilwell and Bradley E. Bishop. Platoons of underwater vehicles. IEEE Control Systems Magazine, 20(6):45-52, December 2000.

27. P. K. C. Wang and F. Y. Hadaegh. Coordination and control of multiple microspacecraft moving in formation. The Journal of the Astronautical Sciences, 44(3):315-355, 1996. 